\title{
Patient-Specific Monitoring and Trend Analysis of Model-based Markers of Fluid Responsiveness in Sepsis: A proof-of-concept animal study
}

\author{
Liam Murphy $^{1}$, Shaun Davidson ${ }^{2}$, J. Geoffrey Chase ${ }^{1}$, Jennifer L. Knopp ${ }^{1}$,Tony Zhou ${ }^{1}$, \\ Thomas Desaive ${ }^{3}$
}

September 11, 2019

\footnotetext{
${ }^{1}$ Department of Mechanical Engineering, University of Canterbury, Christchurch, New Zealand;

${ }^{2}$ Institute of Biomedical Engineering, University of Oxford, England; and ${ }^{3}$ Liege University, Liege, Belgium.
}

\begin{abstract}
Total stressed blood volume $\left(S B V_{T}\right)$ and arterial elastance $\left(E_{a}\right)$ are two a potentially important, clinically applicable metrics for guiding treatment in patients with altered hemodynamic states. Defined as the total pressure generating blood in the circulation, $S B V_{T}$ is a potential direct measurement of tissue perfusion, a critical component in treatment of sepsis. $E_{a}$ is closely related to arterial tone thus provides insight into cardiac efficiency. However, it is not clinically feasible or ethical to measure $S B V_{T}$ in patients, so a three chambered cardiovascular system model using measured left ventricle pressure and volume, aortic pressure and central venous pressure is implemented to identify $S B V_{T}$ and $E_{a}$ from clinical data.

$S B V_{T}$ and $E_{a}$ are identified from clinical data from six (6) pigs, who have undergone clinical procedures aimed at simulating septic shock and subsequent treatment, to identify clinically relevant changes. A novel, validated trend analysis method is used to adjudge clinically significant changes in state in the real-time $E_{a}$ and $S B V_{T}$ traces. Results matched hypothesised increases in $S B V_{T}$ during fluid therapy, with a mean change of $+21 \%$ during initial therapy, and hypothesised decreases during endotoxin induced sepsis, with a mean change of $-29 \%$. $E_{a}$
\end{abstract}


displayed the hypothesised reciprocal behaviour with a mean changes of $-12 \%$ and $+30 \%$ during initial therapy and endotoxin induced sepsis, respectively. The overall results validate the efficacy of $S B V_{T}$ in tracking changes in hemodynamic state in septic shock and fluid therapy.

Keywords - Stressed Blood Volume, Arterial Elastance, Cardiovascular, Fluid Therapy 


\section{Introduction}

Sepsis is a leading cause of intensive care unit (ICU) patient admission, comprising $10 \%$ of US ICU admissions, and making septic shock the 10th ranked cause of death in the US at an estimated cost of $\$ 16.7$ billion and 215,000 lives annually [23], [12]. Patients who display significant cardiovascular dysfunction in sepsis have a mortality rate of approximately $70-90 \%$. There is thus a need for better insight and care to improve outcome and cost.

Sepsis is defined as inappropriate systemic inflammatory response to infection resulting in ischemia, hypotension, multiple organ failure, and potentially death. Treatment of sepsis and septic shock are guided by the Surviving Sepsis campaign, which gives details on recommended treatment guidelines [9]. Until as recently, as 2016, this guide was based on an early goal-directed treatment, which aimed to achieve specified metrics for patient outputs, including mean arterial pressure and central venous pressure [15], through fluid infusions and various drug administrations. The rationale behind this approach is an increase in volume results in increased arterial pressure and sustained tissue perfusion allowing organs to continue normal function. Early goal-directed treatment has recently been shown to deliver no significant improvement in patient well-being [25], when compared to previous guidelines, generating confusion and inconsistent requirements for treatment.

Currently, the surviving sepsis campaign guidelines recommend administrating $30 \mathrm{ml} / \mathrm{kg}$ of fluids in the initial resuscitation phase. However, as approximately only $50 \%$ of septic patients are fluid responsive [3], inappropriate administration can result in increased mortality [22]. Accurately monitoring patient specific response to receiving fluids is therefore critical to appropriate care, but requires insight to patient condition not yet available [4].

Total stressed blood volume, $S B V_{T}$, is defined as the total pressure generating blood volume in the circulation [19]. It thus influences venous return and is a direct potential measure of tissue perfusion. $S B V_{T}$ is the diference between the total blood volume in circulation and the unstressed

volume, where the unstressed volume is the minimum volume required to fill a blood vessel to the point where it exerts a force on the vessel wall [30]. This definition makes $S B V_{T}$ a parameter of 
interest in sepsis patients. $S B V_{T}$ has recently been shown to be a potential index of fluid responsiveness [19],[27] showing a negative correlation between $S B V_{T}$ and cardiac output [27].

$S B V_{T}$ is difficult to measure directly and involves performing several cardiac arrests and vascular fillings. As such it is impractical and unethical to perform this procedure in the ICU [20], and thus the need for a model-based analogue is present. Finally, model based arterial elastance, $E_{a}$, is also considered in this work due to its relation with unstressed volume, its relation to arterial tone lost in septic shock, and the effect a change in this parameter can have on the efficiency of the circulation [13].

A lumped three chambered cardiovascular system (CVS) model, first introduced by Pironet et $a l$, is implemented to develop model-based analogues of total stressed blood volume, $S B V_{T}$, and arterial elastance, $E_{a}$, in porcine subjects with induced septic shock. Real time monitoring of these variables can provide a new insight into the state of the CVS during sepsis. Monitoring $S B V_{T}$ could allow clinicians to move from a 'one size fits all' goal pressure or set volume for fluid infusions to a tailored patient specific treatment 'one method fits all' approach, based on patient specific progression of disease state and response to care [5].

This study aims to show how a three chambered CVS model can be used to monitor model based analogues of $S B V_{T}$ and $E_{a}$. It aims to show how these metrics are effected under simulated septic shock and fluid treatments, giving a first insight into the clinical applicability of these metrics.

\section{Methods}

\subsection{Cardiovascular system model}

The CVS model used in this work is a lumped, three-chambered model previously described by Pironet et al [27],[28],[29], consisting of three elastic chambers connected via three flow resistances. The chambers represent the left ventricle, $l v$, aorta, $a$, and one vena cava, $v c$, while the resistances are indicative of an input, $R_{i}$, and output, $R_{o}$, cardiac resistance and the systemic circulation resistance, $R_{c}$. The model is shown in Figure 1. 




Figure 1: Three-Chamber Model Schematic

The arterial and venous chambers are passive chambers with pressure in the chambers defined:

$$
\begin{array}{r}
P_{a}(t)=E_{a} V_{s, a}(t) \\
P_{v c}(t)=E_{v c} V_{s, v c}(t)
\end{array}
$$

where $E$ is the chamber elastance and $V_{s}$ is the stressed volume of the chamber. Stressed volume is the difference between the total volume and the unstressed volume, $V_{u}$, in the chamber.

The left ventricular chamber is an active chamber described using a time varying pressurevolume relationship [27],[37],[28]:

$$
P_{l v}(t)=e(t) E_{l v} V_{s, l v}(t)
$$

where $E_{l v}$ is the end systolic elastance of the left ventricle, $V_{s, l v}(\mathrm{t})$ is the stressed volume and $e(\mathrm{t})$ is the normalised time-varying function derived following the work of Davidson et al. [7] and detailed in the following section.

As stated, the chambers are linked through flow resistances $R_{i}, R_{o}$, and $R_{c}$ representing the input and output valve, and the systemic circulation resistance, respectively. Flow in the systemic 
circulation is modelled using Ohm's law:

$$
Q_{c}(t)=\frac{P_{a}(t)-P_{v c}(t)}{R_{c}}
$$

where $R_{c}$ is the value of the resistance of the circulation capturing the capillaries and surrounding arterial-venous resistance. Flows in and out of the left ventricle are described by piecewise functions due to flow through the valves only existing if a positive pressure gradient exists.

$$
\begin{gathered}
Q_{i}(t)=\left\{\begin{array}{cl}
\frac{P_{v c}(t)-P_{l v}(t)}{R_{i}} & \text { if } \quad P_{v c}>P_{l v} \\
0 & \text { otherwise }
\end{array}\right. \\
Q_{o}(t)=\left\{\begin{array}{cl}
\frac{P_{l v}(t)-P_{a}(t)}{R_{o}} & \text { if } \quad P_{v l}>P_{a} \\
0 & \text { otherwise }
\end{array}\right.
\end{gathered}
$$

Calculating the flows from measured data and back calculation allows for properties such as viscosity to be incorporated without the need for direct measurement. Finally, the continuity equation is used to give the rate of change of volume for each of the chambers.

$$
\dot{V}_{S, c}(t)=Q_{\text {in }, c}-Q_{o u t, c}
$$

where $Q_{i n, c}$ and $Q_{o u t, c}$ represent the flow in and out of chamber $c(c \in\{a, v c, l v\})$. Summing the rate of change of volume for all three chambers gives the total rate of change of stressed volume for the system. As the system is treated as closed, the volume rate of change for the system is zero, and thus the total stressed blood volume $\left(V_{s, 3}\right)$ is a constant defined:

$$
V_{s, l v}(t)+V_{s, v c}(t)+V_{s, a}(t)=V_{s, 3}(t)
$$

The complete system is thus defined through 8 model parameters $\left(3\right.$ chamber elastances: $E_{l v} ; E_{a}$; and $E_{v c} ; 3$ flow resistances: $R_{i} ; R_{o}$; and $R_{c}$; the cardiac period, $T$; and total stressed blood volume, $\left.V_{s, 3}\right)$, and a cardiac driver function $e(\mathrm{t})$. Measured values for aortic pressure, left ventricle pressure, left ventricle volume and central venous pressure are used to derive all necessary initial estimates for the model parameters. Measured data is also used to construct error metrics corresponding to 
their representative parameters for parameter identification and validation.

\subsection{Parameter Identification}

As some parameters are not sensitive enough to be identified because of their relatively low impact on the error function, a subset selection algorithm is used [29],[28]. Initial values for the model parameters $\left(E_{l v}, E_{a}, E_{v c}, R_{i}, R_{o}, R_{c}\right.$ and $\left.S B V_{T}\right)$ are calculated [28] and passed through the subset selection algorithm, which aims to select the set of parameters to be optimised by the parameter identification method. This selection is completed using the Hessian matrix to evaluate the effect each parameter has on the error vector and selecting a subset which can be optimised. The subset of parameters selected were $\left(\left\{E_{l v}, E_{a}, E_{v c}, R_{i}, R_{c}, S B V_{T}\right\}\right)$. Parameter identification in this work was completed using MATLAB's (The Mathworks, Natick, MA, USA) fmincon function. The selected subset was input to the function which minimized the error sum of squares of the parameters with respect to their measured counterpart. Cardiac period $(T)$ is calculated directly from available pressure waveforms.

\subsection{Driver function model $(e(t))$}

Time varying elastance (TVE) represents the active elastance in the cardiac chambers dictating the heart's contraction and expansion [7],[36]. TVE curves are derived using ventricular pressure and volume waveforms and contain information which can be used to drive cardiovascular models. Intrinsic to TVE curves is information pertaining to the stages of the cardiac cycle, such as filling, isovolumetric contraction, and ejection. This information is vital in CVS models and TVE curves are commonly implemented as driver functions defined:

$$
e(t)=\frac{P_{l v}(t)}{V_{l v}(t)-V_{u}}
$$

where $V_{u}$ is the unstressed volume in the left ventricle and $P_{l v}$ and $V_{l v}$ are the left ventricle pressure and volume, respectively. Previous work [7] has shown baseline $V_{u}$ can be approximated as a fixed percentage of the end-systolic volume $\left(V_{e s}\right)$, which allows an approximation of $V_{u}$ during an initial 
echocardiographic calibration, in which $V_{e s}$ is available [7]. Thus, $V_{u}$ can be expressed [6]:

$$
V_{u}=0.48 \times V_{e s}
$$

The method used to derive the driver function in this work is described by Davidson et al [7] and recreates pressure and volume waveforms using simple physiological assumptions combined with readily available clinical measurements. Continuous measurements of aortic pressure $\left(P_{a}\right)$ and heart rate $(H R)$, typically available in the ICU, in addition to baseline measurements of end-systolic $\left(V_{e s}\right)$ and end-diastolic $\left(V_{e d}\right)$ volumes obtained from echocardiography readings are used to create patient specific TVE curves. Thus, the method enables minimally invasive construction of TVE curves as it removes the need for directly measured $P_{l v}$ and $V_{l v}$, which require highly invasive procedures and additional catheters to obtain. It also avoids the need to directly assume the shape of the curve, as done in previous methods [7], [36]. Finally, since finding $S B V_{T}$ only relies only on aortic (or distal) arterial pressure and central venous pressure, it is non-additionally invasive in an ICU context as it requires no additional catheters than are standard practice use for treating hemodynamically unstable patients. This non-additional-invasiveness ensures a minimally to non- invasive method.

To approximate the $P_{l v}$ waveform the method assumes aortic valve resistance is negligible. As a result, during the majority of systole, one can equate $P_{l v}$ to $P_{a}$, separated by some phase lag due to the ventricle being located directly upstream of the aorta [7],[33],[35]. The $P_{a}$ waveform gives little information about the left ventricle during diastole, but left ventricular behaviour in this region is known to be relatively passive [7]. As such, left ventricular pressure during diastole can be modelled as a function consisting of two exponentials, an exponential increase for the beginning of contraction and an exponential decay to a fixed value to model ventricular relaxation. Using 
these principles, the left ventricular pressure can be estimated from aortic pressure as follows [7]:

$$
\begin{aligned}
& t_{1}=t\left({\frac{d P_{a}}{d t_{\max }}}_{n}\right. \\
& t_{2}=t\left({\frac{d P_{a}}{d t}}_{\text {min }}\right)_{n} \\
& t_{3}=0.62 t_{2}+0.38 t_{4} \\
& t_{4}=t\left({\frac{d P_{a}}{d t_{\max }}}_{n+1}\right. \\
& P_{l v}= \begin{cases}P_{a}\left(t_{1}+\delta<t<t_{2}+\delta\right) & t_{1}<t<t_{2} \\
6+\left(P_{a\left(t_{2}\right)}-6\right) e^{-17.5\left(t-t_{2}\right)} & t_{2}<t<t_{3} \\
P_{l v}\left(t_{3}\right)+\left(P_{a}\left(t_{4}\right)-P_{l v}\left(t_{3}\right)\right) e^{37.5\left(t-t_{4}\right)} & t_{3}<t<t_{4}\end{cases}
\end{aligned}
$$

where $\delta=0.008$ s. Timings detailed in Equations 11 - 14 indicate key positions of an aortic wave used for the reconstruction of the left ventricle pressure wave. These positions are illustrated in Figure 2(a) and show how information can be translated from measured waveforms to a simulated waveform.

The $V_{l v}$ waveform is recreated using a piecewise sine wave with a $90^{\circ}$ phase shift in conjunction with 6 pieces of information derived from the aortic pressure waveform, 3 timings, $t_{1}, t_{2}, t_{3}$, and 3 volumes, $V_{d}, V_{e d}, V_{e s}$, associated with diastole and systole. Previous work has shown end-systolic volume and end-diastolic volume can be expressed:

$$
\begin{array}{r}
V_{e s}=\frac{P_{D N}}{E_{c} \times H R^{3}}+V_{d} \\
V_{e d}=V_{e s}+S V
\end{array}
$$

where $P_{D N}$ is the aortic pressure at the dicrotic notch and $H R$ and $E_{c}$ represent heart rate and an elastance coefficient, respectively. $H R$ and $E_{c}$ are used to define $E_{e s}$ and the cubic term in Equation 16 allows for the best accuracy while maintaining low model complextity [8]. Finally, the left-ventricular volume waveform can be expressed: 


$$
V_{l v}(t)= \begin{cases}\left(V_{e d}\right)_{n}+\left(\left(V_{e s}\right)_{n}-\left(V_{e d}\right)_{n}\right) \sin \left(\frac{\pi\left(t-t_{1}\right)}{2\left(t_{2}-t_{1}\right)}\right) & t_{1}<t<t_{2} \\ \left(V_{e s}\right)_{n}+\left(\left(V_{e d}\right)_{n+1}-\left(V_{e d}\right)_{n}\right)\left(\frac{1}{2} \cos \left(\frac{\pi\left(t-t_{2}\right)}{\left(t_{3}-t_{2}\right)}\right)-\frac{1}{2}\right) & t_{2}<t<t_{3}\end{cases}
$$

Where:

$$
\begin{array}{r}
t_{1}=t\left(P_{a_{\text {min }}}\right)_{n} \\
t_{2}=t\left(P_{D N}\right)_{n} \\
t_{3}=t\left(P_{a_{\text {min }}}\right)_{n+1}
\end{array}
$$

Timings detailed in Equations 19 - 21 indicate key positions on the $n^{\text {th }}$ aortic wave used for the reconstruction of the left ventricle volume wave. These positions are illustrated in Figure 2 (b) and show how information can be translated from measured waveforms to a simulated waveform.



(a)

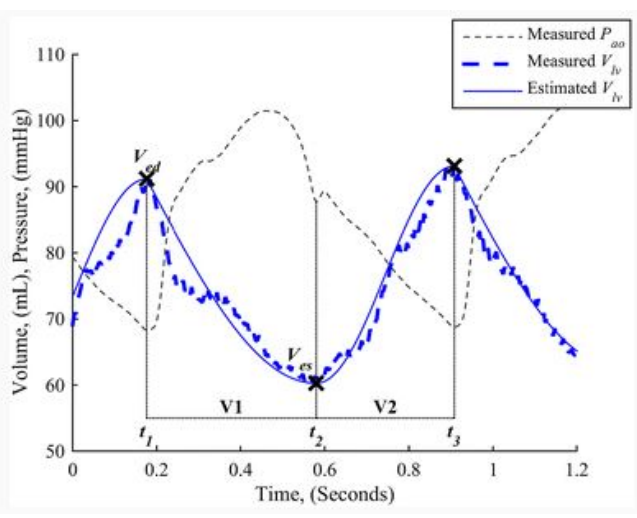

(b)

Figure 2: Estimated $P_{l v}$ and $V_{l v}$ derived from invasively measured $P_{a o}$

\subsection{State Classification and Trend Monitoring}

State classification in this work was performed using a modified version of the algorithm in [39] to identify clinically relevant changes in $S B V_{T}$ and $E_{a}$ during the experiment. This method calculates the mean over an entire experiment or data series to establish a global mean. A 5 to 10 minute centered rolling average of the data points is then calculated to remove the effects of any outliers 
in the data, which may have occurred during data collection [39]. This average was selected based on clinically relevant time frames defining a clinically relevant state. The rolling average curves, an example of which is depicted in Figure 3, are then compared to the global mean, and the intercepts between the two curves (indicated by the blue circles) are noted. Each intercept between the rolling average and the global mean is considered to determine if they are clinically relevant. If the time between state changes is less than the rolling average time, this section of data is added to the previous state and a new state average is calculated. Changes in state averages can then be investigated to determine their correlation with any clinical procedures or events occurring during that stage of the experiment.

The state analysis in this work performs two iterations to capture information, which may be otherwise missed due to the length of the experiment and the inherent intra- and inter- subject variability in subjects [11]. The second iteration is calculated the same, but uses each first iteration states (purple lines) in place of the global average. Variations of the rolling average about these states are considered with each intercept (purple circles) being checked for clinically relevant state changes, as shown in Figure 3. The second iteration better differentiates rapid state changes as it is considering the mean of certain subsections of the experiment and is also clinically defined. The peak in Figure 4 between the first two original intercepts with the global mean is an example of when fluctuations with duration less than that used for the rolling average occur, resulting in the first and second iteration generating the same state.

Maas et al defined an increase in cardiac output $>12 \%$ as a positive response to a $500 \mathrm{ml}$ fluid therapy [19], clinically defining a state change. Thus, as this is a first study into changes in $S B V_{T}$ and due to certain physiological similarities between $S V$ and $S B V_{T}$, a change in $S B V_{T}$ or $E_{a}$ of $\pm 10 \%$ is considered significant in this work for all state changes and defines a change of state in response to fluid therapy and endotoxin infusion.

\subsection{Experimental Data}

The experimental protocol was approved by the Ethics Committee for the use of animals at the University of Liege, Belgium. Six (6) pure Pietrain pigs were anaesthetised and mechanically 


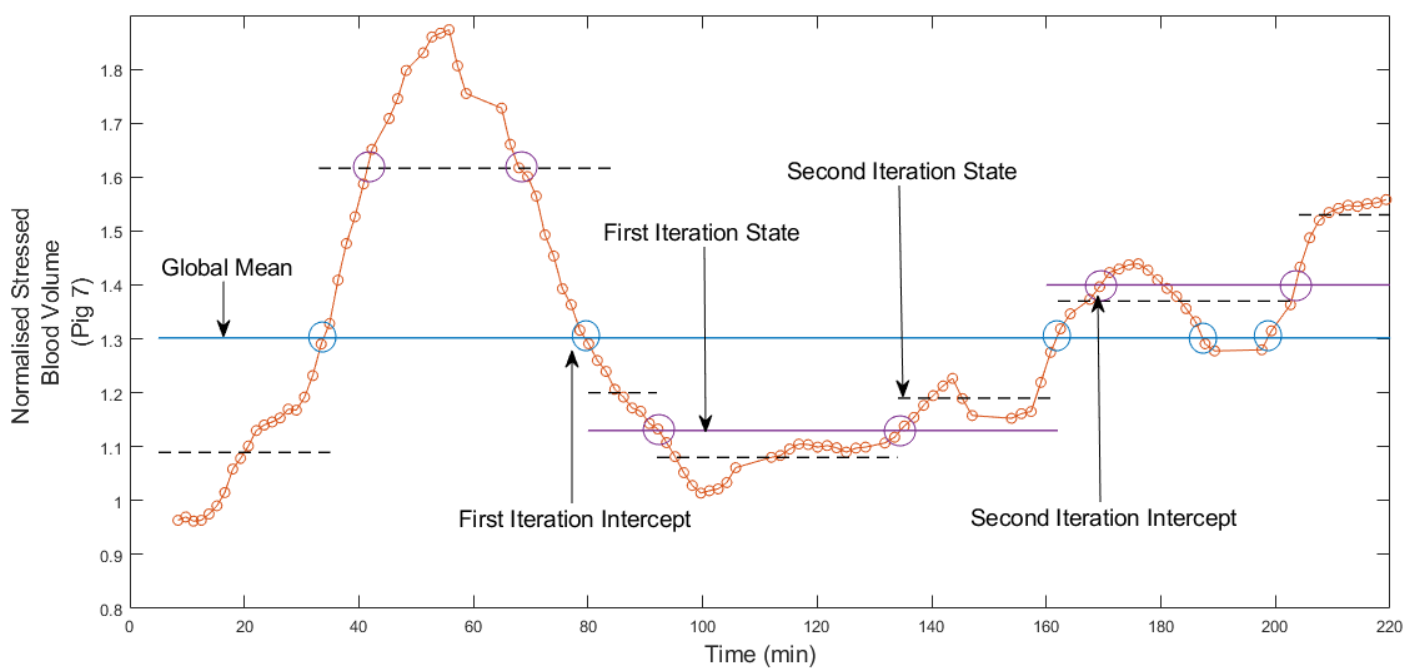

Figure 3: Example of how the two stage state analysis is conducted with circled components detailing the intercepts which are analysed to determine any state changes.

ventilated. Septic shock was then induced in the subjects via a one off infusion of endotoxin (lipopolysaccharide from E. Coli, $0.5 \mathrm{mg} / \mathrm{kg}$ infused over $30 \mathrm{~min}$ ). Pre-endotoxin infusion, a 500 $\mathrm{mL}$ saline solution is first administered over $30 \mathrm{~min}$ simulating fluid resuscitation therapy. Aortic pressure in the subjects is continually measured via a catheter with a sampling rate of $250 \mathrm{~Hz} . P_{l v}$ and $V_{l v}$ are also continually measured at a rate of $250 \mathrm{~Hz}$ via an admittance pressure volume catheter inserted into the left ventricle via an apical stab. Measured $S V$ is also compared to reinforce and validate the trends and responses seen in the model-based $S B V_{T}, E a$ metrics. In this work only Pigs 1, 2, 4, 6, 7 and 8 are used due to errors in recording of data for the remaining subjects making the data unsuitable for this model. Faults such a catheter being suspected of pressing up against vessel walls and continuous shifts in data made Pigs 3 and 5 unreliable for this work.

\subsection{Analyses}

The outputs of this work displays a time series of modelled $S B V_{T}, E_{a}$ and directly measured catheter values of $S V$. As shown in Figure 4, trends are observed through state changes, which detail when a clinically defined change in the metrics occur and are depicted by horizontal dashed lines. Output data is analysed to establish if displayed trends matched that hypothesised. It is expected that: 
- A fluid infusion will result in a noticeable rise in $S B V_{T}$ ideally greater than $10 \%$, indicating a positive fluid response. Null or lesser changes would indicate fluid unresponsiveness.

- Inversely, it is expected $E_{a}$ will decrease in response to a positive response to fluid infusion.

- Trends seen during or soon after sepsis is induced, via endotoxin infusion, are expected to be the reverse of fluid infusions with $S B V_{T}$ dropping and $E_{a}$ rising. 


\section{Results}

Figures 5 and 6 show the identified $S B V_{T}$ and $E_{a}$ trajectories during an initial fluid resuscitation therapy, induction of sepsis, and subsequent fluid infusions for all pigs. Also included in Figures 5 and 6 is catheter measured $S V$, which is commonly used in investigations of fluid responsiveness and is also physiologically similar to $S B V$, thus provides a meaningful comparison. For clarity, data points on the figures show results for every 100th heart beat. Vertical lines are indicative of the start and end of each the various procedures with the colour indicating which procedure is being performed. Figure 4 shows the iterative state analysis to identify trends in subject responses for a single subject. State averages are represented by horizontal dashed lines and represent when a clinically relevant change in the modelled parameter has occurred. The two staged state identification process employed ensures smaller, more rapid, responses are not overlooked.

Tables 1 - 3 detail the numerical state average changes for the corresponding parameters. Results generally matched hypothesised behaviours, with $S V B_{T}$ having mean increase of $+21 \%$ during initial filling and falling with a mean of $-29 \%$ in endotoxin induced sepsis. $E_{a}$ shows similar results in reverse, as hypothesised. For subjects that survived the endotoxin infusion (Pigs 2, 6 and 7), further fluid therapies were administered. The results are also displayed in Tables 1 - 3. Pigs which have not displayed any fluctuation around the global average are displayed as a dash (-) while deceased Pigs are denoted with a 'D', indicating no further data follows. Values in red text represent where a change in the metric has occurred but is below the $\pm 10 \%$ threshold.

Tables 1 - 3 show the collated results for iterative state analysis across all subjects. It details the percentage increase or decrease in response to the associated clinical procedure. A dash implies no state change was observed either due to no effect being seen or the response to the procedure was smaller than the clinically specified $10 \%$ limit and thus not considered clinically relevant [19]. 



Figure 4: Example result of State Analysis for each variable of Pig 7. Horizontal lines indicate state changes while vertical lines represent timings of fluid therapies: First (Red); Second (Blue); Third (Black); and endotoxin infusion (Green). 

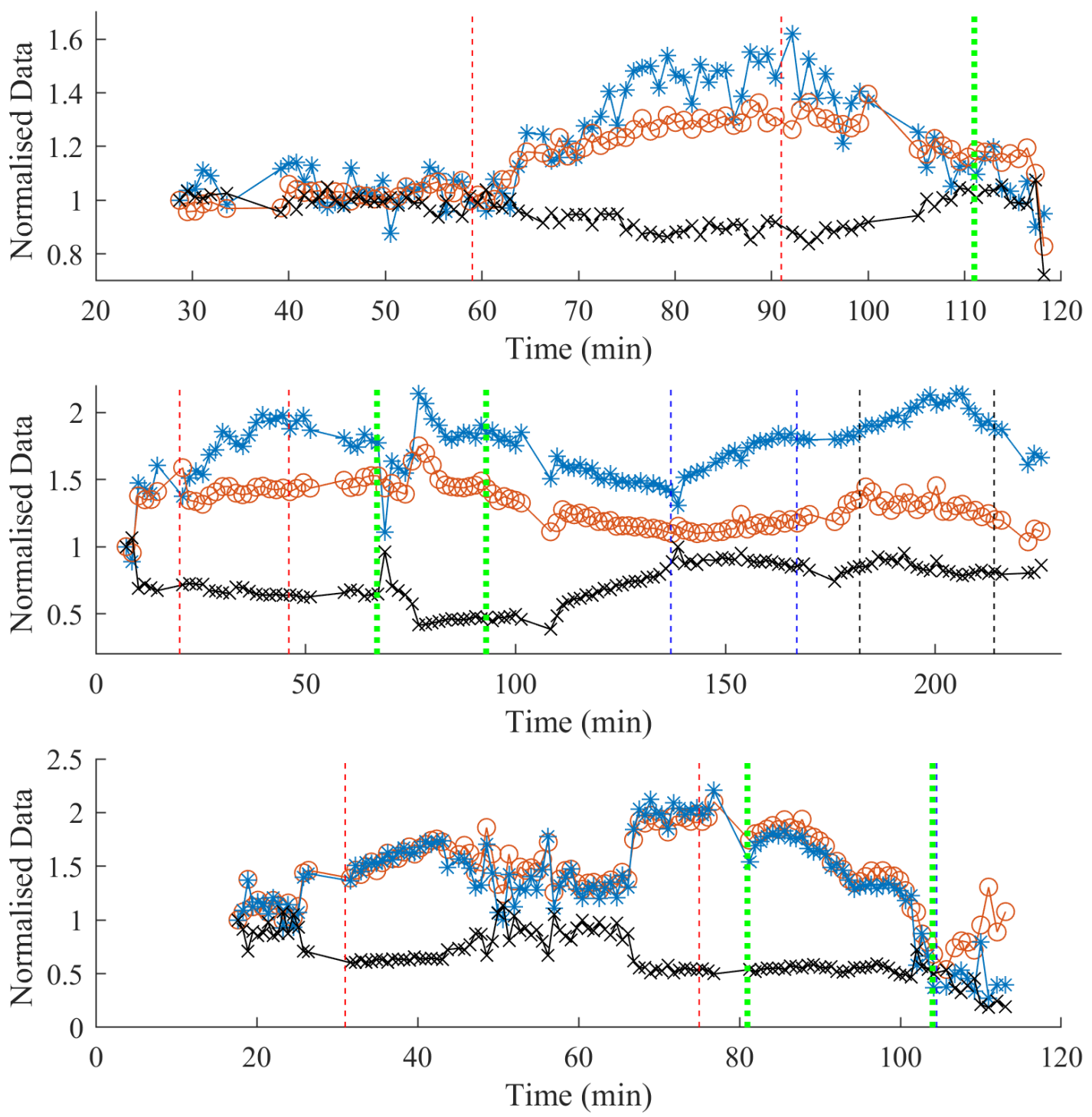

Figure 5: Changes in model based analogues of $S B V_{T}(\circ)$ and $E_{a}(\mathrm{x})$ and measured $S V(*)$ with respect to various clinical procedures of Pigs 1 (TOP), 2 (MIDDLE) and 4 (BOTTOM). Vertical lines represent timings of fluid therapies: First (Red); Second (Blue); Third (Black); and endotoxin infusion (Green). 



Figure 6: Changes in model based analogues of $S B V_{T}(\circ)$ and $E_{a}(\mathrm{x})$ and measured $S V$ (*) with respect to various clinical procedures of Pigs 6 (TOP), 7 (MIDDLE) and 8 (BOTTOM). Vertical lines represent timings of fluid therapies: First (Red); Second (Blue); Third (Black); and endotoxin infusion (Green). 


\begin{tabular}{|l|c|c|c|c|}
\hline & $S B V_{T}$ (Fill 1) & $S B V_{T}$ (Endo) & $S B V_{T}$ (Fill 2) & $S B V_{T}$ (Fill 3) \\
\hline Pig 1 & $+22 \%$ & - & $\mathrm{D}$ & $\mathrm{D}$ \\
\hline Pig 2 & $+4.6 \%$ & $-23 \%$ & $+17 \%$ & $-10 \%$ \\
\hline Pig 4 & $+22 \%$ & $-39 \%$ & $\mathrm{D}$ & $\mathrm{D}$ \\
\hline Pig 6 & $+11 \%$ & $-26 \%$ & $-12 \%$ & - \\
\hline Pig 7 & $+42 \%$ & $-33 \%$ & $+10 \%$ & $+15 \%$ \\
\hline Pig 8 & $+24 \%$ & $-55 \%$ & $\mathrm{D}$ & $\mathrm{D}$ \\
\hline MEAN & $\mathbf{+ 2 1 \%}$ & $\mathbf{- 2 9 \%}$ & $\mathbf{+ 1 3 \%}$ & $\mathbf{+ 8 . 3 \%}$ \\
\hline
\end{tabular}

Table 1: Relative change in $S B V_{T}$ during clinical procedures.

\begin{tabular}{|l|c|c|c|c|}
\hline & $E_{a}$ (Fill 1) & $E_{a}$ (Endo) & $E_{a}$ (Fill 2) & $E_{a}$ (Fill 3) \\
\hline Pig 1 & $-9.0 \%$ & - & $\mathrm{D}$ & $\mathrm{D}$ \\
\hline Pig 2 & $-29 \%$ & $+81 \%$ & $-4.6 \%$ & $-7.8 \%$ \\
\hline Pig 4 & $-19 \%$ & - & $\mathrm{D}$ & $\mathrm{D}$ \\
\hline Pig 6 & - & $+24 \%$ & $+25 \%$ & $+10 \%$ \\
\hline Pig 7 & $-16 \%$ & $+15 \%$ & $+14 \%$ & $+15 \%$ \\
\hline Pig 8 & - & $+60 \%$ & $\mathrm{D}$ & $\mathrm{D}$ \\
\hline MEAN & $\mathbf{- 1 2 \%}$ & $\mathbf{+ 3 0 \%}$ & $\mathbf{+ 8 . 3 \%}$ & $\mathbf{+ 5 . 7 \%}$ \\
\hline
\end{tabular}

Table 2: Relative change in $E_{a}$ during clinical procedures.

\begin{tabular}{|l|c|c|c|c|}
\hline & SV (Fill 1) & SV (Endo) & SV (Fill 2) & SV (Fill 3) \\
\hline Pig 1 & $+25 \%$ & - & D & D \\
\hline Pig 2 & $+20 \%$ & $-19 \%$ & $+19 \%$ & $+8.5 \%$ \\
\hline Pig 4 & $+20 \%$ & $-52 \%$ & D & D \\
\hline Pig 6 & $+22 \%$ & $-32 \%$ & $+26 \%$ & - \\
\hline Pig 7 & $+25 \%$ & $-32 \%$ & $+4.3 \%$ & $+14 \%$ \\
\hline Pig 8 & $+22 \%$ & $-60 \%$ & D & D \\
\hline MEAN & $\mathbf{+ 2 2 \%}$ & $\mathbf{- 3 3 \%}$ & $\mathbf{+ 1 6 \%}$ & $+\mathbf{7 . 5 \%}$ \\
\hline
\end{tabular}

Table 3: Relative change in $S V$ during clinical procedures. 


\section{Discussion}

This work introduces two model based analogues for Total Stressed Blood Volume $\left(S B V_{T}\right)$ and arterial Elastance $\left(E_{a}\right)$ derived using the presented three chamber model. The model used measured values for aortic pressure, left ventricle pressure and volume and central venous pressure to derive $S B V_{T}$ and $E_{a}$. This work deems these parameters significant due to their representation of tissue perfusion and arterial tone, respectively. However, direct measurements of these values are unable to be conducted in an ICU resulting in the need for model based analogues.

Positive responses to fluid therapy have previously been clinically defined by a change in cardiac output of greater than $12 \%$ [19] and is the desired outcome of a fluid infusion. Due to variability in subjects and the differing procedures performed, this work considers a state average change of $2 \times \pm 5 \%$ to be clinically significant. This multiplication is due to the two part iteration of the state analysis. Administering fluids aims to increase cardiac output by increasing the total volume, and thus an increase in $S B V_{T}$ is expected. If no increase in $S B V_{T}$ is detected from fluid filling it would suggest some CVS dysfunction and potentially increased vascular permeability leading to tissue hypoperfusion. Equally, no response may be due to the subject not being hypovolemic, resulting in excess fluid, which can appear in the lungs causing ventilation difficulties [22],[21],[32].

\subsection{Stressed Blood Volume}

During the initial fluid therapy, a state average increase of $S B V_{T}$ occurs in all subjects, with an average increase of $20.9 \%$. This result indicates these subjects experienced a positive response to the fluid infusion. Due to the fluid infusions being administered pre-endotoxin, positive responses are expected, as the subjects are still considered healthy, and may also explain why such substantial increases are present due to no CVS dysfunction being present yet to cause fluid loss. Similarly, Pigs 1, 6 and 7 display a decrease in $S B V_{T}$ towards the end of this procedure, which may be due to the fluid therapy being conducted on a healthy subject, where excess fluid is unnecessary, or less necessary, and therefore fluid overload may have occurred. In this circumstance, it is possible a hypervolemic state is induced forcing fluid into the interstitial space and seen as a drop in $S B V_{T}$ [38]. 
With the exception of Pig 1, Table 1 shows all subjects experience a state average decrease of $S B V_{T}$ in response to the endotoxin infusion, as hypothesised and expected. A mean state decrease in $S B V_{T}$ of $-26 \%$ is seen in response to endotoxin, indicating significant complications in the cardiovascular system. Pig 1 also shows a decrease in this section, but insufficient survival time for this subject means a state change in $S B V_{T}$ is not found, although it's evident in the end of Figure 5. As septic shock is induced fluid is lost from the circulation due to increased vascular permeability [24], [14]. The loss in total volume would result in a drop in blood pressure and $S B V_{T}$, which are both characteristic of septic shock [21], [14],[18],[31] and evident in this experiment.

Post endotoxin infusion, 3 subjects, Pigs 2, 6 and 7, received further fluid therapies allowing for monitoring of parameters during a simulated clinical response to septic shock. During the first post-endotoxin fluid therapy Pigs 2 and 7 saw a state increase in $S B V_{T}$ indicating a positive response to treatment. The effects of treatment in both pigs can be seen to hold until the second post-endotoxin fluid therapy, where there is a disparity in the responses. While Pig 7 displays an initial increase in $S B V_{T}$ during filling, a drop midway through is indicative of a natural response to fluid overload. Fluid overload commonly causes a drop in $S V$ and $\mathrm{CO}$ and exacerbates the effects of sepsis on the cardiovascular system [21],[1],[2],[17]. Thus, a corresponding drop in $S B V_{T}$ would also be expected given this condition. Pig 2 displays a similar trend to Pig 7 with a decrease in $S B V_{T}$ part way through filling, but with no initial increase in observed.

Smaller responses in $S B V_{T}$ are seen for Pigs 2 and 6 which may be due to the starting point of the experiment and the prior data available for analysis. Pigs 2, 6 and 8 have an initial fluid therapy shortly after the start of the experiment due to positioning issues with equipment rendering prior recordings difficult to use. Due to the nature of the state change analysis and the relatively short time before fluid therapy, a smaller response may occur.

\subsection{Arterial Elastance}

Pig 6 is the only subject to have no discernible positive response to fluid therapy post endotoxin. Both the second and third fluid therapies post-endotoxin cause a slight increase in $S B V_{T}$, but the effects are brief, and, in both cases, begin to drop before the end of the treatment. This outcome 
indicates the subject was not fluid responsive in either case, which is likely due to a severe reaction to endotoxin and/or the subject is simply not fluid responsive.

$E_{a}$ during initial fluid therapy either decreased or saw no change in state averages for all subjects. As this therapy is first performed on a healthy subject it is expected there would be little effect on the elastance as endotoxin has yet to initiate any issues in the system or arterial tone. A decrease in $E_{a}$ may also be in response to the volume administered, with the blood vessels relaxing to accommodate this extra fluid. If sepsis were affecting the properties of the arterial system causing an increase in stiffness, a rise in $E_{a}$ during or soon after the endotoxin infusion would be expected. Due to the variability in biological systems it is expected subjects will respond to endotoxin differently [10]. Thus, the time during and immediately following endotoxin infusion are considered.

In general, $E_{a}$ showed a state increase in response to endotoxin infusion, as expected. A mean increase of $36 \%$ over the 5 Pigs (Pigs 2, 4, 6, 7 and 8) to survive past endotoxin administration was found. The 2 Pigs that do not display a state change in $E_{a}$ in response to endotoxin (Pigs 2 and 4) had severe reactions and died immediately after (Pig 4) or during (Pig 2) the procedure. Maintaining appropriate blood pressure in subjects with sepsis is of vital importance and stiffening of the arterial/venous tone is one means of regulating this pressure in hypovolemic states. The two pigs which had a severe reaction may have done so due to an inability to increase $E_{a}$ to maintain adequate blood pressure, as a sign of the body fighting back. Early, rapid death of Pigs 2 and 4 meant insufficient time was available for a state change to be calculated [39].

During the two post-endotoxin fluid therapies, Pigs 6 and 7 showed state average increases in $E_{a}$. These results are consistent with the response seen in $S B V_{T}$ during this time, again suggesting and supporting the conclusion these subjects were not fluid responsive. Pig 2 also displays a trend in $E_{a}$ consistent with changes seen in $S B V_{T}$. During both post-endotoxin fluid therapies, $E_{a}$ experiences a state drop, indicating the fluid is having a positive and expected effect.

In conjunction with the two parameters identified from the model $\left(S B V_{T}, E_{a}\right)$, catheter measured $S V$ was also monitored for the corresponding heartbeats as a form of validation. As expected, 
trends in $S V$ closely resemble those of $S B V_{T}$, but also provide additional information of interest. All subjects see a state average increase during the initial fluid with a mean increase of $22.5 \%$, which is again expected due to the CVS still considered healthy and no infection present. Pigs 1, 6 and 7 show a decrease in $S V$ at the end of filling, reinforcing the judgement that fluid overload is occurring in these subjects. Significant decreases in $S V$ are seen in all subjects, with the exception of Pig 1, which died prior to the completion of endotoxin. A mean state average decrease of $39 \%$ across the surviving pigs validates the fact induced sepsis has a severe impact on $S V$, as seen clinically [23],[12].

Post-endotoxin fluid infusions show $S V$ is generally more sensitive to fluid therapy than $S B V_{T}$ and displays a faster response to treatment. All surviving subjects had increases in $S V$ from the first filling post-endotoxin, but Pig 7 displayed only a small increase which appeared to maintain $S V$ at the current level, rather than provide any significant increase. Pig 2 experiences a positive response in $S V$ from the second post-endotoxin filling, which is in contrast to the negative response in $S B V_{T}$. However, while $S V$ is more sensitive, $S B V_{T}$ may be the better clinical measure if performance improvement is the primary goal of fluid therapy.

\subsection{Limitations}

One of the biggest limitations of the model presented in this work is it only encompasses the systemic circulation and omits the pulmonary circulation [27],[28]. The underlying assumption here is the systemic and pulmonary circulations can be treated separately, which is not physiologically accurate. Only considering the systemic circulation also means there is no ventricular interaction. In the body, the heart is enclosed a fibrous membrane called the pericardium, which causes a compressive force applied to the left ventricle when the right undergoes filling. With only one circulation being considered this interaction cannot be accounted for, though it is typically small except in pulmonary embolism [33],[34].

Physiologically the left ventricle and parts of the arterial and venous systems are located in the thoracic chamber, which results in a thoracic pressure being applied to part of the systemic circulation. Again, as only one circulation is accounted for in this model it is difficult to accurately account for this pressure. Thoracic pressure effects the right ventricle differently to the left, and 
this effect cannot be properly recreated here. Anatomically, parts of the passive arterial and venous chambers are also located in the thoracic chamber making the resulting effect difficult to account for. However, this influence likely only applies a small consistent bias, where the trends over treatment are potentially more clinically important.

A noticeable difference in the model to true anatomy is the absence of an atrium, which provideds part of the pressure required to fill the ventricle. It has not yet been shown that the time varying elastance theory can be applied to the atria as it is done to the ventricle. Other authors have developed alternate methods to represent the atria such as the multi-scale model [26]. However, the large number of parameters required for such methods to work cannot be identified using the limited data clinically available, making it unsuitable to apply in this work. Although the atria is excluded from the model, model parameters can be adjusted to account for this absence. Filling of the left ventricle is represented as a passive process thus, $R_{i}$, the parameter describing the flow resistance into the ventricle, will likely be lower than reality to compensate for no atria, providing a totally passive equivalent filling.

Finally, this study is limited by the small sample size of experimental data available. Although limited in number, the porcine data used in this study provided accurate information to run and validate the model, including left ventricle volume, which is not typically available in the ICU. Such animal studies are intensive, time consuming, and costly, thus the number can be limited. However, the quality of data available for validation is very high, and unavailable in human subjects. Thus, this study and data provide a foundation of work, which can be confidently built on to investigate trends in a larger population of human subjects, while directing it towards a clinical setting.

\subsection{Conclusion}

The work presented in this paper used measured aortic, central venous and left ventricular pressure and volume to provide information for the model. The model presented in this work outputs a set of parameters two of which, $S B V_{T}$ and $E_{a}$, are potentially important metrics of fluid responsiveness. Although some of this measured data is currently available in the ICU, measured left ventricular volume is typically not accessible and pressures are not usually measured in the direct 
locations used here. Future work would employ emerging methods [16] to capture $S V$ from diastole pressure measures and thus use currently available ICU data, avoiding the need for any new procedures/devices to be implemented. 


\section{References}

[1] Sean M Bagshaw, Patrick D Brophy, Dinna Cruz, and Claudio Ronco. Fluid balance as a biomarker: impact of fluid overload on outcome in critically ill patients with acute kidney injury. Critical care, 12(4):169, 2008. doi: 10.1186/cc6948.

[2] Liam Byrne and Frank Haren. Fluid resuscitation in human sepsis: time to rewrite history? Annals of intensive care, 7(1):4, 2017. doi: 10.1186/s13613-016-0231-8.

[3] Fabio Cavallaro, Claudio Sandroni, and Massimo Antonelli. Functional hemodynamic monitoring and dynamic indices of fluid responsiveness. Minerva anestesiologica, 74(4):123-135, 2008.

[4] Maurizio Cecconi, Daniel De Backer, Massimo Antonelli, Richard Beale, Jan Bakker, Christoph Hofer, Roman Jaeschke, Alexandre Mebazaa, Michael R Pinsky, Jean Louis Teboul, et al. Consensus on circulatory shock and hemodynamic monitoring. task force of the european society of intensive care medicine. Intensive care medicine, 40(12):1795-1815, 2014. doi: 10.1007/s00134-014-3525-z.

[5] J Geoffrey Chase, Aaron J Le Compte, Jean-Charles Preiser, Geoffrey M Shaw, Sophie Penning, and Thomas Desaive. Physiological modeling, tight glycemic control, and the icu clinician: what are models and how can they affect practice? Annals of intensive care, 1(1):11, 2011. doi: 10.1186/2110-5820-1-11.

[6] Shaun Davidson, Chris Pretty, Antoine Pironet, Thomas Desaive, Nathalie Janssen, Bernard Lambermont, Philippe Morimont, and J Geoffrey Chase. Minimally invasive estimation of ventricular dead space volume through use of frank-starling curves. PloS one, 12(4):e0176302, 2017. doi: 10.1371/journal.pone.0176302.

[7] Shaun Davidson, Chris Pretty, Antoine Pironet, Shun Kamoi, Joel Balmer, Thomas Desaive, and J. Geoffrey Chase. Minimally invasive, patient specific, beat-by-beat estimation of left ventricular time varying elastance. BioMedical Engineering OnLine, 16(1):42, Apr 2017. ISSN 1475-925X. doi: 10.1186/s12938-017-0338-7. URL https://doi.org/10.1186/s12938-017-0338-7. 
[8] Shaun M Davidson, Chris Pretty, Shun Kamoi, Joel Balmer, Thomas Desaive, and J Geoffrey Chase. Real-time, minimally invasive, beat-to-beat estimation of end-systolic volume using a modified end-systolic pressure-volume relation. IFAC-PapersOnLine, 50(1):5456-5461, 2017. doi: 10.1016/j.ifacol.2017.08.1082.

[9] R Phillip Dellinger, Mitchell M Levy, Andrew Rhodes, Djillali Annane, Herwig Gerlach, Steven M Opal, Jonathan E Sevransky, Charles L Sprung, Ivor S Douglas, Roman Jaeschke, et al. Surviving sepsis campaign: international guidelines for management of severe sepsis and septic shock, 2012. Intensive care medicine, 39(2):165-228, 2013. doi: 10.1007/S00134-0122769-8.

[10] Jennifer L Dickson, Cameron A Gunn, and J Geoffrey Chase. Humans are horribly variable. Int J Clin Med Imaging, 1(2):1-1000142, 2014.

[11] JL Dickson, CA Gunn, and JG Chase. Clinical \& medical imaging. International Journal, 1 (2):1000142, 2014.

[12] Konstantinos Drosatos, Anastasios Lymperopoulos, Peter Johannes Kennel, Nina Pollak, P Christian Schulze, and Ira J Goldberg. Pathophysiology of sepsis-related cardiac dysfunction: driven by inflammation, energy mismanagement, or both? Current heart failure reports, 12(2):130-140, 2015. doi: 10.1007/s11897-014-0247-z.

[13] Fabio Guarracino, Rubia Baldassarri, and Michael R Pinsky. Ventriculo-arterial decoupling in acutely altered hemodynamic states. Critical care, 17(2):213, 2013. doi: 10.1186/cc12522.

[14] Hori Hariyanto, Corry Quando Yahya, Monika Widiastuti, Primartanto Wibowo, and Oloan Eduard Tampubolon. Fluids and sepsis: changing the paradigm of fluid therapy: a case report. Journal of medical case reports, 11(1):30, 2017. doi: 10.1186/s13256-016-1191-1.

[15] Michael D Howell and Andrew M Davis. Management of sepsis and septic shock. Jama, 317 (8):847-848, 2017. doi: 10.1001/jama.2017.0131.

[16] Shun Kamoi, Christopher Pretty, Joel Balmer, Shaun Davidson, Antoine Pironet, Thomas Desaive, Geoffrey M Shaw, and J Geoffrey Chase. Improved pressure contour analysis for esti- 
mating cardiac stroke volume using pulse wave velocity measurement. Biomedical engineering online, 16(1):51, 2017. doi: 10.1186/s12938-017-0341-z.

[17] Diana J Kelm, Jared T Perrin, Rodrigo Cartin-Ceba, Ognjen Gajic, Louis Schenck, and Cassie C Kennedy. Fluid overload in patients with severe sepsis and septic shock treated with early-goal directed therapy is associated with increased acute need for fluid-related medical interventions and hospital death. Shock (Augusta, Ga.), 43(1):68, 2015. doi: 10.1001/jama.2016.0288.

[18] Aseem Kumar, Joseph E Parrillo, Anand Kumar, et al. Clinical review: myocardial depression in sepsis and septic shock. Critical care, 6(6):500, 2002. doi: 10.1186/cc1822.

[19] Jacinta J Maas, Michael R Pinsky, Leon P Aarts, and Jos R Jansen. Bedside assessment of total systemic vascular compliance, stressed volume, and cardiac function curves in intensive care unit patients. Anesthesia \& Analgesia, 115(4):880-887, 2012. doi: 10.1213/ANE.0b013e31825fb01d.

[20] Sheldon Magder and Benoit De Varennes. Clinical death and the measurement of stressed vascular volume. Critical care medicine, 26(6):1061-1064, 1998.

[21] Manu LNG Malbrain, Niels Van Regenmortel, Bernd Saugel, Brecht De Tavernier, PieterJan Van Gaal, Olivier Joannes-Boyau, Jean-Louis Teboul, Todd W Rice, Monty Mythen, and Xavier Monnet. Principles of fluid management and stewardship in septic shock: it is time to consider the four d's and the four phases of fluid therapy. Annals of intensive care, 8(1):66, 2018. doi: 10.1186/s13613-018-0402-x.

[22] P Marik and Rinaldo Bellomo. A rational approach to fluid therapy in sepsis. BJA: British Journal of Anaesthesia, 116(3):339-349, 2015. doi: 10.1093/bja/aev349.

[23] MW Merx and C Weber. Sepsis and the heart. Circulation, 116(7):793-802, 2007. doi: 10.1161/circulationaha.106.678359.

[24] Xavier Monnet, Paul E Marik, and Jean-Louis Teboul. Prediction of fluid responsiveness: an update. Annals of intensive care, 6(1):111, 2016. doi: 10.1186/s13613-016-0216-7. 
[25] Paul R Mouncey, Tiffany M Osborn, G Sarah Power, David A Harrison, M Zia Sadique, Richard D Grieve, Rahi Jahan, Sheila E Harvey, Derek Bell, Julian F Bion, et al. Trial of early, goal-directed resuscitation for septic shock. New England Journal of Medicine, 372(14): 1301-1311, 2015. doi: 10.1056/NEJMoa1500896.

[26] Antoine Pironet, Pierre C Dauby, Sabine Paeme, Sarah Kosta, J Geoffrey Chase, and Thomas Desaive. Simulation of left atrial function using a multi-scale model of the cardiovascular system. PloS one, 8(6):e65146, 2013. doi: 10.1371/journal.pone.0065146.

[27] Antoine Pironet, Pierre C Dauby, J Geoffrey Chase, Shun Kamoi, Nathalie Janssen, Philippe Morimont, Bernard Lambermont, and Thomas Desaive. Model-based stressed blood volume is an index of fluid responsiveness. IFAC-PapersOnLine, 48(20):291-296, 2015. doi: 10.1016/j.ifacol.2015.10.154.

[28] Antoine Pironet, Thomas Desaive, J. Geoffrey Chase, Philippe Morimont, and Pierre C. Dauby. Model-based computation of total stressed blood volume from a preload reduction manoeuvre. Mathematical Biosciences, 265:28 - 39, 2015. ISSN 0025-5564. doi: https://doi.org/10.1016/j.mbs.2015.03.015. URL http://www.sciencedirect.com/science/article/pii/S0025556415000826.

[29] Antoine Pironet, Thomas Desaive, Pierre C Dauby, J Geoffrey Chase, and Paul D Docherty. Parameter identification methods in a model of the cardiovascular system. IFACPapersOnLine, 48(20):366-371, 2015. doi: 10.1016/j.ifacol.2015.10.167.

[30] CARL F Rothe. Mean circulatory filling pressure: its meaning and measurement. Journal of Applied Physiology, 74(2):499-509, 1993. doi: 10.1152/jappl.1993.74.2.499.

[31] Christopher W Seymour, Vincent X Liu, Theodore J Iwashyna, Frank M Brunkhorst, Thomas D Rea, André Scherag, Gordon Rubenfeld, Jeremy M Kahn, Manu Shankar-Hari, Mervyn Singer, et al. Assessment of clinical criteria for sepsis: for the third international consensus definitions for sepsis and septic shock (sepsis-3). Jama, 315(8):762-774, 2016. doi: 10.1001/jama.2016.0288.

[32] João M Silva, Amanda Maria RibasRosa de Oliveira, Fernando Augusto Mendes Nogueira, 
Pedro Monferrari Monteiro Vianna, Marcos Cruz Pereira Filho, Leandro Ferreira Dias, Vivian Paz Leão Maia, Cesar de Souza Neucamp, Cristina Prata Amendola, Maria José Carvalho Carmona, et al. The effect of excess fluid balance on the mortality rate of surgical patients: a multicenter prospective study. Critical Care, 17(6):R288, 2013. doi: 10.1186/cc13151.

[33] Bram W Smith, J Geoffrey Chase, Roger I Nokes, Geoffrey M Shaw, and Tim David. Velocity profile method for time varying resistance in minimal cardiovascular system models. Physics in Medicine $\&$ Biology, 48(20):3375, 2003. doi: 10.1088/0031-9155/48/20/008/meta.

[34] Christina Starfinger, Christopher E Hann, J Geoffrey Chase, Thomas Desaive, Alexandre Ghuysen, and Geoffrey M Shaw. Model-based cardiac diagnosis of pulmonary embolism. Computer methods and programs in biomedicine, 87(1):46-60, 2007. doi: 10.1016/j.cmpb.2007.03.010.

[35] David Stevenson, James Revie, J Geoffrey Chase, Christopher E Hann, Geoffrey M Shaw, Bernard Lambermont, Alexandre Ghuysen, Philippe Kolh, and Thomas Desaive. Algorithmic processing of pressure waveforms to facilitate estimation of cardiac elastance. Biomedical engineering online, 11(1):28, 2012. doi: 10.1186/1475-925X-11-28.

[36] David Stevenson, James Revie, J Geoffrey Chase, Christopher E Hann, Geoffrey M Shaw, Bernard Lambermont, Alexandre Ghuysen, Philippe Kolh, and Thomas Desaive. Beat-to-beat estimation of the continuous left and right cardiac elastance from metrics commonly available in clinical settings. Biomedical engineering online, 11(1):73, 2012. doi: 10.1186/1475-925X11-73. URL http://dx.doi.org/10.1186/1475-925X-11-73.

[37] Hiroyuki Suga, Kiichi Sagawa, and Artin A Shoukas. Load independence of the instantaneous pressure-volume ratio of the canine left ventricle and effects of epinephrine and heart rate on the ratio. Circulation research, 32(3):314-322, 1973.

[38] Jean-Louis Vincent and Michael R Pinsky. We should avoid the term "fluid overload", 2018.

[39] Tony Zhou, Jennifer Knopp, Christopher JD McKinlay, Gregory D Gamble, Jane E Harding, J Geoffrey Chase, CHYLD Study Group, et al. Glycaemic state analysis from continuous 
glucose monitoring measurements in infants. IFAC-PapersOnLine, 51(27):276-281, 2018. doi: 10.1016/j.ifacol.2018.11.629. 